\title{
Invariance and randomness in the Nash program for coalitional games
}

\author{
Nir Dagan ${ }^{\mathrm{a}, *}$, Roberto Serrano \\ a Department of Economics, Universitat Pompeu Fabra, Ramon Trias Fargas 25-27, 08005 Barcelona, Spain \\ ${ }^{\mathrm{b}}$ Department of Economics, Brown University, Providence, RI 02912, USA \\ Received 29 July 1997; accepted 15 September 1997
}

\begin{abstract}
Introducing physical outcomes in coalitional games we note that they and social choice problems are equivalent (implying that so are the theory of implementation and the Nash program). This clarifies some misunderstandings (in regard to invariance and randomness), sometimes found in the Nash program. (c) 1998 Elsevier Science S.A.
\end{abstract}

Keywords: Nash program; Implementation; Scale invariance; Ordinal invariance; Randomness

JEL classification: $\mathrm{C} 70$

\section{Introduction}

The standard definition of a coalitional game does not include physical outcomes, but only feasible utility profiles. This abstract framework may sometimes lead to basic misunderstandings. The purpose of this note is to dispel two such misconceptions, found in the Nash program. Generalizing the model in Serrano (1997), we extend the definition of a coalitional game to include physical outcomes. This will prove helpful to our purpose.

If players are Von Neumann-Morgenstern expected utility maximizers, the different properties of invariance of a utility scale to transformations must play a central role. We present two results. Both are simple corollaries of invariance and they are well understood (especially the first one) by the practitioners of the abstract theory of implementation. Unfortunately, the same cannot be said for the Nash program, both in its folklore and in its printed material. One could attribute this to the "black box" of the characteristic function, entangled with the view that "the Nash program is not implementation."

The first result asserts that a coalitional solution concept can be arrived at from the noncooperative theory only if it is scale invariant. By the same token, if a solution is not scale invariant its normative appeal is also very restricted, since an uninformed planner cannot implement it. Essentially, if there is

\footnotetext{
*Corresponding author. Tel.: 001401 8633836; fax: 001401 8631970; e-mail: robert_serrano@brown.edu
} 
a noncooperative game related to such a solution, the game must change with the underlying environment (see Bossert and Tan, 1995 for a game related to the egalitarian bargaining solution).

We shall consider a solution concept to coalitional games as being independent of any randomization if it can be implemented by a strategic game and a strategic equilibrium concept that do not include any random element. Our second result says that if a solution concept is independent of randomizations, it must be ordinally invariant (like the core, bargaining sets or Walrasian equilibria). This implies that major solution concepts in coalitional games (e.g. the Nash bargaining solution, the NTU-Shapley value) can be derived strategically only by considering the possibility of random outcomes: either chance moves, mixed strategies, or pure strategy equilibrium refinements based on trembles must be part of the analysis. Mechanisms that support these solution concepts cannot be criticized for introducing random devices, since they are essential to the implementation results. We can dispel now numerous comments (received in seminars over the years) present in the folklore, like "the fair coin again?," "an implementation of the Shapley value would be much nicer without randomness, which already gives it away," or "Can't you think of more realistic and natural mechanisms? When have you seen people randomizing in the real world?" Thus, our second result stresses the major role that risk plays in much of the theory of coalitional games.

\section{The model}

Usually, coalitional games are represented by characteristic functions:

Definition. A characteristic function game $(N, V)$ consists of

1. a set of players $N=\{1,2, \ldots, n\}$;

2. a characteristic function $V$ that assigns to every nonempty set of players $S \subset N$ a set $V(S)$ of real valued utility profiles (that the members of $S$ may reach).

In the above standard definition physical outcomes are not specified. We consider the following (more general) notion:

Definition. A coalitional game $\Gamma=\left(N, X,\left\{u_{i}\right\}_{i \in N}\right)$ consists of:

1. a set of players $N=\{1,2, \ldots, n\}$;

2. a pure outcome function $X$ that assigns to every nonempty set of players $S \subset N$ a set of $S$-outcomes $X(S)$ that the members of $S$ may reach. The function $X$ satisfies: if $X(S) \neq \varnothing$, then there exists a partition $\left\{T_{1}, \ldots, T_{k}\right\}$ of $N S$ with $X\left(T_{i}\right) \neq \varnothing$ for all $i=1, \ldots, k$; and there exists a coalition $Q$ with $X(Q) \neq \varnothing$.

3. for each player $i \in N$, a payoff function $u_{i}$ that assigns a real number $u_{i}(x)$ to every outcome $x \in \cup X(S)$, where the union is taken over all coalitions $S$ that contain $i$.

An outcome of a coalitional game is a partition of the set of players and an $S$-outcome for each 
coalition $S$ in the partition. We denote by $X_{N}(\Gamma)$ the set of all outcomes of a coalitional game $\Gamma$, and by $X_{N}^{*}(\Gamma)$ the set of all probability distributions on $X_{N}(\Gamma)$. Since we want to distinguish between random and nonrandom outcomes in the sequel, we introduce these distinctions here.

Note that our assumptions on the outcome function $X$ imply that the set $X_{N}(\Gamma)$ summarizes all the information in $X$, and that $X_{N}(\Gamma) \neq \varnothing$. Also, the definition restricts attention to c-games (Shubik, 1984, pp. 130-131).

Definition. Let $\Omega$ be a class of coalitional games. A pure solution $\psi$ on $\Omega$ is a correspondence that assigns to each coalitional game $\Gamma \in \Omega$ a set $\psi(\Gamma) \subset X_{N}(\Gamma)$.

Definition. A solution $\psi$ on $\Omega$ is a correspondence that assigns to each coalitional game $\Gamma \in \Omega$ a set $\psi(\Gamma) \subset X_{N}^{*}(\Gamma)$.

Solution concepts which are defined for characteristic function games can be adapted into our framework by assigning to each outcome of the characteristic function game a nonempty set of outcomes of the coalitional game.

Another important class of problems found in the literature is gathered under the name of social choice problems.

Definition. A social choice problem $\Pi=\left(N, A,\left\{u_{i}\right\}_{i \in N}\right)$ consists of:

1. a set of players $N=\{1,2, \ldots, n\}$;

2. a nonempty set of alternatives $A$;

3. for each player $i \in N$, a payoff function $u_{i}$ that assigns a real number $u_{i}(a)$ to every alternative $a \in A$.

There is a natural equivalence between social choice problems and coalitional games. For every social choice problem $\left(N, A,\left\{u_{i}\right\}_{i \in N}\right)$ assign the coalitional game $\left(N, X,\left\{u_{i}\right\}_{i \in N}\right)$ defined by $X(N)=A$, $X(S)=\varnothing$ if $S \neq N$. Conversely, we can associate a social choice problem to each coalitional game by defining $A=X_{N}(\Gamma)$.

An immediate consequence of the equivalence established in the previous paragraph is that the abstract theory of implementation and the Nash program for coalitional games should be viewed as the same research agenda. See Serrano (1997) on this point.

\section{Results on invariance}

By emphasizing the physical structure of coalitional games, one may be able to reach certain general conclusions that were difficult to draw from the characteristic function. Our results on invariance below are simple examples of this.

Definition. Let $\Gamma=\left(N, X,\left\{u_{i}\right\}_{i \in N}\right)$ be a coalitional game. A coalitional game $\Gamma^{\prime}=\left(N, X,\left\{w_{i}\right\}_{i \in N}\right)$ is a positive affine transformation of $\Gamma$ if for all $i \in N$ there exist real numbers $\alpha_{i}>0$ and $\beta_{i}$ such that for all outcomes $x$ in which $i$ is involved in $w_{i}(x)=\alpha_{i} u_{i}(x)+\beta_{i}$. 
Definition. A solution $\psi$ on $\Omega$ is scale invariant if for every two games $\Gamma, \Gamma^{\prime} \in \Omega$, such that $\Gamma^{\prime}$ is a positive affine transformation of $\Gamma$, then $\psi(\Gamma)=\psi\left(\Gamma^{\prime}\right)$.

Definition. Let $\Gamma=\left(N, X,\left\{u_{i}\right\}_{i \in N}\right)$ be a coalitional game. A coalitional game $\Gamma^{\prime}=\left(N, X,\left\{w_{i}\right\}_{i \in N}\right)$ is an order preserving transformation of $\Gamma$ if for all $i \in N$ and for all outcomes $x$ and $x^{\prime}$ in which $i$ is involved in, $w_{i}(x)>w_{i}\left(x^{\prime}\right)$ if and only if $u_{i}(x)>u_{i}\left(x^{\prime}\right)$.

Definition. A pure solution $\psi$ to a class of coalitional games $\Omega$ is ordinally invariant if for every two games $\Gamma, \Gamma^{\prime} \in \Omega$, such that $\Gamma^{\prime}$ is an order preserving transformation of $\Gamma$, then $\psi(\Gamma)=\psi\left(\Gamma^{\prime}\right)$.

Next we present analogous definitions for strategic games, as our conclusions are relevant for the Nash program. Our results below hold also for games in extensive form, and only for the sake of brevity, we omit the definitions.

Definition. A strategic game $G=\left[N,\left(S_{i}\right)_{i \in N},\left(u_{i}\right)_{i \in N}\right]$ consists of:

1. A set of players $N=\{1,2, \ldots, n\}$; and for all $i \in N$

2. A set of pure strategies $S_{i}$;

3. A payoff function $u_{i}$ that assigns a real number $u_{i}(s)$ to every profile $s \in \Pi_{i \in N} S_{i}$.

Definition. The mixed extension of a strategic game $G=\left[N,\left(S_{i}\right)_{i \in N},\left(u_{i}\right)_{i \in N}\right]$ is a strategic game $G^{*}=\left[N,\left(S_{i}^{*}\right)_{i \in N},\left(u_{i}^{*}\right)_{i \in N}\right]$, such that for all $i \in N$ :

1. $S_{i}^{*}$ is the set of probability distributions over $S_{i}$ (mixed strategies of $G$ ); and

2. $u_{i}^{*}$ assigns to every profile of mixed strategies $s^{*} \in \Pi_{i \in N} S_{i}^{*}$ the expected utility that corresponds to the probability distribution over pure strategies and to the payoffs $u_{i}$.

We can distinguish between two types of equilibrium concepts in strategic games. One takes into account only considerations of the strategic game, and the other considers also the mixed extension. Clearly, pure strategy Nash equilibrium belongs to the former type, and mixed strategy Nash equilibrium belongs to the latter. However, there are some selections from pure strategy Nash equilibrium (e.g., trembling hand perfect) that take into account considerations of the mixed extension. The distinction between the two types of equilibrium concepts cannot be made by using their definitions, as these may be adapted to incorporate the mixed extension. In order to make the distinction formal we introduce the concepts of invariance in strategic games as well.

In all strategic equilibrium concepts, agents are assumed to choose their actions with accordance to their preferences over the relevant set of outcomes. This implies that the theory should be insensitive to alternative utility representations of the same ordinal preferences. In the case where the mixed extension of the game is considered, the preference order is defined over the mixed outcomes, thus under the expected utility hypothesis it is required that positive affine transformations of the utility function over the pure outcomes would not change the set of equilibria. When the equilibrium concept does not take into account the mixed extension, any order preserving transformation of the utility function over pure outcomes should not change the set of equilibria. First we define the notions of equilibrium concepts. 
Definition. An equilibrium concept $f$ over a class of strategic games $\Sigma$ is a correspondence that assigns to every strategic game $G=\left[N,\left(S_{i}\right)_{i \in N},\left(u_{i}\right)_{i \in N}\right] \in \Sigma$ a set $f(G) \subset \Pi_{i \in N} S_{i}^{*}$.

Definition. A pure strategy equilibrium concept $f$ over a class of strategic games $\Sigma$ is an equilibrium concept for which $f(G) \subset \Pi_{i \in N} S_{i}$ for all $G \in \Sigma$.

Now, we define invariance notions.

Definition. Let $G=\left[N,\left(S_{i}\right)_{i \in N},\left(u_{i}\right)_{i \in N}\right]$ be a strategic game. The game $G^{\prime}=\left[N,\left(S_{i}\right)_{i \in N},\left(w_{i}\right)_{i \in N}\right]$ is a positive affine transformation of $G$ if for all $i \in N$ there exist real numbers $\alpha_{i}>0$ and $\beta_{i}$ such that for all pure strategy profiles $s w_{i}(s)=\alpha_{i} u_{i}(s)+\beta_{i}$.

Definition. An equilibrium concept $f$ over a class of strategic games $\Sigma$ is scale invariant if for every two games $G, G^{\prime} \in \Sigma$ such that $G^{\prime}$ is a positive affine transformation of $G$, it is satisfied that $f(G)=f\left(G^{\prime}\right)$.

Definition. Let $G=\left[N,\left(S_{i}\right)_{i \in N},\left(u_{i}\right)_{i \in N}\right]$ be a strategic game. The game $G^{\prime}=\left[N,\left(S_{i}\right)_{i \in N},\left(w_{i}\right)_{i \in N}\right]$ is an order preserving transformation of $G$ if for all $i \in N$ and for all pure strategy profiles $s$ and $s^{\prime}$, $w_{i}(s)>w_{i}\left(s^{\prime}\right)$ if and only if $u_{i}(s)>u_{i}\left(s^{\prime}\right)$.

Definition. A pure-strategy equilibrium concept $f$ over a class of strategic games $\Sigma$ is ordinally invariant if for every two games $G, G^{\prime} \in \Sigma$ such that $G^{\prime}$ is an order preserving transformation of $G$, it is satisfied that $f(G)=f\left(G^{\prime}\right)$.

The class of ordinally invariant equilibrium concepts includes pure strategy Nash equilibrium, pure strategy subgame perfect equilibrium (and its stationary refinements), pure undominated strategies, iterative elimination of dominated actions, among others.

Now we turn to connect the definitions of invariance in coalitional and strategic games, which will have simple implications for the Nash program.

Definition. Let $\Omega$ be a set of coalitional games all of which share the same pair $(N, X)$. A mechanism with respect to a pair $(N, X)$ consists of two components:

1. a pair $\left[N,\left(S_{i}\right)_{i \in N}\right]$ with the same set of players and a pure strategy set for each of them,

2. a function $m: \prod_{i \in N} S_{i} \rightarrow X_{N}^{*}(\Gamma)$.

Definition. Let $\Omega$ be a set of coalitional games all of which share the same pair $(N, X)$. A mechanism $\left[N,\left(S_{i}\right)_{i \in N}, m\right]$ implements $\psi$ on $\Omega$ by $f$ if for all $\Gamma \in \Omega f\left(\left[N,\left(S_{i}\right)_{i \in N},\left(u_{i}^{m}\right)_{i \in N}\right]\right)=\psi(\Gamma)$, where $u_{i}^{m}(s)=u_{i}^{*}(m(s))$.

Result 1. If a mechanism implements a coalitional solution $\psi$ on $\Omega$ by a scale invariant equilibrium concept, then $\psi$ is scale invariant.

Given the above definitions, the easy proof is left to the reader.

Therefore, if we consider interesting classes of bargaining problems, the egalitarian solution, which 
prescribes a weakly Pareto efficient outcome that gives the same utility to each of the players, can never be arrived at from the strategic approach to bargaining. This casts doubt on Thomson's (Thomson, 1994, p. 1242) assertion that this solution is (together with Nash's and Kalai and Smorodinsky's) one of the three most important solutions to bargaining problems.

Bossert and Tan (1995) provide an arbitration game whose Nash equilibria yield the egalitarian bargaining solution. Their arbitration game form changes with the utility functions when one considers the class of all bargaining problems. Alternatively, Result 1 implies that the domain of utility profiles allowed in their game (if we fix the strategy sets) is such that the egalitarian solution is scale invariant on this domain. Actually, our second result shows that their domain is even more restrictive.

Result 2. If a mechanism implements a coalitional solution $\psi$ on $\Omega$ by an ordinally invariant equilibrium concept, and the range of $\mathrm{m}$ is a subset of $X_{N}(\Gamma)$, then $\psi$ is a pure ordinally invariant solution.

Again the easy proof is left to the reader.

In light of this result, and in contrast to some opinions in the folklore, it is not surprising that the implementations of the Shapley value (Gul, 1989; Winter, 1994; Hart and Mas-Colell, 1996), of the Nash bargaining solution (Nash, 1953; Binmore et al., 1986, extended to multilateral bargaining problems by Chae and Yang, 1994 and Krishna and Serrano, 1996), of the kernel (Serrano, forthcoming), of the Kalai-Smorodinsky solution (Moulin, 1984), among others, all include chance moves in one way or another. The solution concepts that are implemented by these mechanisms when applied to NTU games are scale invariant, but not ordinally invariant. Also, for some classes of coalitional games, it is easy to adapt the mechanisms above based on the characteristic function to our framework with physical outcomes.

Returning to Bossert and Tan (1995) arbitration game, considered as the division of a dollar. Notice that the hypotheses of Result 2 are met (as the authors use pure strategy Nash equilibria, and all the possible outcomes of their game do not require randomization), thereby implying the following: the class of bargaining problems for which the strategy sets of the game are fixed must be such that the egalitarian solution is ordinally invariant. That is, their domain is so restrictive that the egalitarian solution yields the same division of the dollar for all problems. We therefore disagree with Thomson (1994), p. 1276), who considers this work as an implementation result.

The reader may be puzzled by Result 2 and the "support" of the Nash solution by Binmore et al. (1986) when it is based on the bargainers' time preferences (Rubinstein, 1982). Although pure strategy subgame perfect equilibrium is ordinally invariant, it should be noted that an ordinal change of the preferences over the division of the dollar in a given period constitutes a real change in the bargainers" time preferences. Thus, we use the word "support" in quotation marks, as the set of pure outcomes of the pair $\left[N,\left(S_{i}\right)_{i \in N}\right]$ is not a subset of the set of pure outcomes of the coalitional game, but an extended set that includes time contingent outcomes that do not appear in the coalitional game. In contrast, in the interpretation of the discount factor as the probability of breakdown, the two sets of feasible pure outcomes (that of the coalitional game and that of the mechanism) coincide, but then of course the chance moves appear explicitly in the strategic situation. We find, like Osborne and Rubinstein (1990), section 4.4, that the interpretation of the Nash bargaining solution with risky outcomes is more natural than the one based on time preferences. 
Thus, risk appears in the theory of coalitional games as one of its fundamental components, in the sense that many central solutions (such as the NTU Shapley values, pure bargaining solutions) rely heavily on it. This fact had been stated for bargaining theory in the work of Nash (1950), but it had not been emphasized as much in general coalitional games. We conclude that randomness in game theory is not just a trick to convexify best responses in order to prove existence of equilibrium. Randomness is a necessary condition to implement many solutions to coalitional games. It is interesting to note that randomness is viewed in the abstract theory of implementation as a way to enhance the set of correspondences that can be implemented (see, for example, Abreu and Matsushima, 1992).

\section{Acknowledgements}

Dagan acknowledges the hospitality of Brown University. Serrano acknowledges the hospitality of Universitat Pompeu Fabra and the financial support of grant SAB95-0186 from Spain's Ministry of Education and Science. ONCE also provided research support.

\section{References}

Abreu, D., Matsushima, H., 1992. Virtual implementation in iteratively undominated strategies: Complete information. Econometrica 60, 993-1008.

Binmore, K., Rubinstein, A., Wolinsky, A., 1986. The Nash bargaining solution in economic modelling. Rand Journal of Economics 17, 176-188.

Bossert, W., Tan, G., 1995. An arbitration game and the egalitarian bargaining solution. Social Choice and Welfare 12, 29-41.

Chae, S., Yang, J.-A., 1994. An $n$-person pure bargaining game. Journal of Economic Theory 62, 86-102.

Gul, F., 1989. Bargaining foundations of the Shapley value. Econometrica 57, 81-95.

Hart, S., Mas-Colell, A., 1996. Bargaining and value. Econometrica 64, 357-380.

Krishna, V., Serrano, R., 1996. Multilateral bargaining. Review of Economic Studies 63, 61-80.

Moulin, H., 1984. Implementing the Kalai-Smorodinsky bargaining solution. Journal of Economic Theory 33, $32-45$.

Nash, J.F., 1950. The bargaining problem. Econometrica 18, 155-162.

Nash, J.F., 1953. Two person cooperative games. Econometrica 21, 128-140.

Osborne, M.J., Rubinstein A., 1990. Bargaining and Markets, Academic Press, San Diego, CA.

Rubinstein, A., 1982. Perfect equilibrium in a bargaining model. Econometrica 50, 97-109.

Serrano, R., 1997. A comment on the Nash program and the theory of implementation. Economics Letters, forthcoming.

Serrano, R., forthcoming. Reinterpreting the kernel. Journal of Economic Theory.

Shubik, M., 1984. Game Theory in the Social Sciences: Concepts and Solutions, 1st paperback ed. M.I.T. Press, Cambridge, MA.

Thomson, W., 1994. Cooperative models of bargaining. In: Aumann, R.J., Hart, S. (Eds.), Handbook of Game Theory with Economic Applications, Vol. 2. North-Holland, Amsterdam.

Winter, E., 1994. The demand commitment bargaining and snowballing of cooperation. Economic Theory 4, $255-273$. 